\title{
SIMULATION DRIVEN PROCESSING FUNCTION DEVELOPMENT, OFFERING AND OPERATION
}

\author{
Dr. Magnus Karlberg, Asc. Prof. \\ Luleå University of Technology \\ Luleå, Sweden
}

\author{
Dr. Magnus Löfstrand Ass. Prof. \\ Luleå University of Technology \\ Luleå, Sweden
}

\author{
Dr. Daniel Marjavaara \\ LKAB \\ Kiruna, Sweden
}

\author{
Prof. T. Staffan Lundström \\ Luleå University of Technology \\ Luleå, Sweden
}

\begin{abstract}
In today's industry, functional provision is becoming more and more important, necessitating increased simulation support. In this paper, the objective is to present a modeling and simulation approach for simulation-driven design (SDD) to support function development. The scope of this paper is simulation support for developing hardware equipment used in processing industry. The research is founded on industrial needs identified through two parallel interview-based studies in the Swedish process industry. Both companies explore doing business with functional products rather than hardware, in scenarios where the responsibility for and availability of the functions may remain with the service provider. One as-is and one future (to-be) scenario are presented. A decomposition of a general processing function (applicable to both companies) describes how the companies transfer machine input to output specifications. The decomposition includes customer and provider value and the paper demonstrates, as part of the results and based on the SDD approach, how that value may be increased through evaluation and prioritization. Additionally, the SDD approach shows that it is possible to identify a set of solutions which meet the specified requirements, supporting evaluation and prioritization of business offers and activities.
\end{abstract}

Keywords: Simulation Driven Design; Functional Products; Product Development; Computer Aided Engineering

\section{INTRODUCTION}

In today's increasingly globalized world, functional provision rather than hardware provision is becoming more and more important. [1] introduce Functional Products (FP) as integrated systems comprising hardware and support services. In general,
FP is an emerging area of interest related to engineering product development. Functional Products consist of Hardware, Software and Services and may be developed for the purpose of providing a function while not necessarily passing ownership of the hardware to the customer $[2,3,4]$. In this context, this paper refers to two parallel studies at two companies in the Swedish process industry. One company is in the pulp and paper industry while the other is in mining. The two companies do not currently have any business relationship. The industrial interest motivating the two companies to partake in the herein reported research is to investigate how possible business regarding provision/procurement of function rather than hardware will affect the company. The functional product approach is a response to a necessity for business-to-business collaborators to gain economy-of-scale partnerships in the extended enterprise and ultimately to be able to develop competitive offers, as discussed by [4] and [1]. Seen from a provider point of view, functional development and provision is of interest for the following reasons:

- competition on the aftermarket can be reduced

- markets which would otherwise be inaccessible without a partnership can be accessed

- long-term customer relationships can be built and maintained

These points are partly based on the fact that a function provider, building on a thorough knowledge of proprietary hardware and customer-use cases, may optimize system delivery function in a way which is generally not possible for a customer. 
Seen from a service customer company view, in addition to decreasing the risks associated with procurement of large, newly developed installations, two reasons for adopting a function procurement approach are:

- lower capital investment

- increased availability of the desired function

Related work with the purpose of supporting availability, [5] considered how firms behave when they face uncertainties arising from both support costs and product performance. [6] present two ways of supporting designers through predicting effects of design changes: probabilistic prediction of the effects of change and visualization of change propagation through product connectivities. Furthermore, [7] introduced previewing tools to facilitate the process of generating, displaying, navigating and evaluating multiple, potential future states simultaneously.

One of the main challenges for this study is how to conduct simulations when developing, offering and operating a functional product. In a benchmark report [8] the Aberdeen group states that manufacturers "...must develop more products that are more complex for their customers..." and that they "...must get to market on time." In order to meet these demands, one recommendation from the Aberdeen group is to "...perform more simulations of product performance in the design phase". However, traditionally, in product development, simulations have rather been used to verify that certain requirements are fulfilled [9]. If not, the designers present a new solution, which is also verified by simulations. This "trial and error procedure" iterates until all requirements are deemed to be fulfilled. Then, the product development process can continue with the construction of a physical prototype that is tested in order to validate the obtained simulation results. This kind of product development methodology has shown to be both expensive and time-consuming and may even prohibit innovation [10]. As the simulation software has developed (especially CAE tools), new methods for how to use simulations in the product development process have been presented. For instance, [11] state that simulation software should be well integrated with the design tools. [12] defines a simulation-driven design (SDD) process as "... a design process where the major functions and related processes are verified and optimised with simulations on computer-based product models." Utilization of such an SDD process on a float-plane development is described by [13]. Furthermore, [14] describes an integrated SDD process where different CAE tools are connected to different stages of a product development process. [15] brings these ideas another step forward by introducing simulations as a design driver rather than just a verifying tool. [16] claim that "...simulationdriven design implies analysis to provide insight for synthesis", which means that goals have to be specified for the simulations.
Since such goals can be contradictory, techniques for multiobjective optimization will be needed. [17] present a multiobjective approach applied in the design of a turbo generator rotor system. [18] show a similar multi-objective approach in combination with surrogate-based optimization analysis for computationally expensive problems. [19] conducted multiobjective optimization simulation of an aircraft based on surrogates and multi-physics simulations. [20] use multiobjective optimization to support simulation-driven synthesis, thus aiding designers. [21] states that a simulation-driven rather than a simulation-verified approach will enable engineers to achieve simulation-driven design by designers. [22] emphasizes that the SDD approach can "...support dialogues with customers, stimulate creation of new concepts and provide guidance towards more optimised designs, especially in early development stages...". When it comes to simulation in a functional product context, [23] presents an approach for how to conduct work process simulations. Such simulations result in better knowledge of company development capacity earlier than before, thus allowing shorter reaction time to changes in the business domain.

So far, little research has been done on how to use simulations in the process industry when the business agreement concerns function-based offers (i.e. processing functions) rather than a traditional hardware offer. Here, we consider the functional offer and how that offer affects the design of the hardware. The Software and Service parts of the functional offer are not within the scope of this particular paper. Hence, the objective for this paper is to propose a SDD methodology suitable for use when developing, offering and operating hardware for processing functions.

\section{RESEARCH APPROACH}

The authors have a good understanding of the studied companies' processes. For the research project reported here, this has enabled good access to the studied process and corresponding activities and has also allowed the authors access to background information as well as knowledge concerning company structures and responsibilities.

One of the authors of this paper has worked in close collaboration with the company in the pulp and paper industry, doing research for seven years. Another of the authors has worked in a similar manner in collaboration with the company involved in mining.

Initially, a workshop was carried out with the aim of identifying and agreeing on which subject areas are most important regarding simulations of functions for the two companies 
studied. Through the discussions, three areas were identified; vision and businesses, product development practices and simulation methodologies. The data collection carried out in the research work consisted of a set of ten interviews and corresponding interview notes based on open-ended, semistructured interviews [24]. The interviews were carried out with five interviewees at the pulp and paper company and five interviewees at the mining company. The interviewees were mainly people with backgrounds in the area of research and development and some of them have experience in business development. At the mining company, two interviewees were general managers of process technology, one interviewee was manager of energy/environment process technology and two interviewees were research engineers in energy/environment process technology.

At the pulp and paper company, one interviewee was general manager, RTD, two were project managers, RTD, one was a general manager, Sales and one a mechanical specialist, RTD.

Five additional interviews were carried out when representatives from both companies attended on the same occasion where the same subjects were harmonized. When the data collection was finished, a feedback workshop was carried out with representatives from the university and the two companies. Representatives from the two companies found it plausible that the result presented in Chapter 6 is a suitable approach given the as-is situation presented in Chapter 3, the scenario in Chapter 4 and the decomposition in Chapter 5.

Analysis of the interview material was carried out through correlation of interviewee statements with official company documents and retrospective project documentation. While the data analysis was carried out, further development of the function decomposition was considered by the industrial partners. The iterative process of data analysis and the resulting decomposition was carried out eight times. Through the data analyses, a set of results was identified, which is presented in Chapter 3, below. The above-discussed data gathering and analysis has further resulted in a developed to-be scenario for functional procurement and provision, which is presented in Chapter 4.

\section{THE AS-IS SITUATION}

Although the two companies studied are active in different branches of industry, their industrial processes and, to a degree, their business processes, are similar. In both cases, raw material with certain specifications enters a processing hardware system. The processing hardware then creates a specified output, as illustrated in Figure 1.

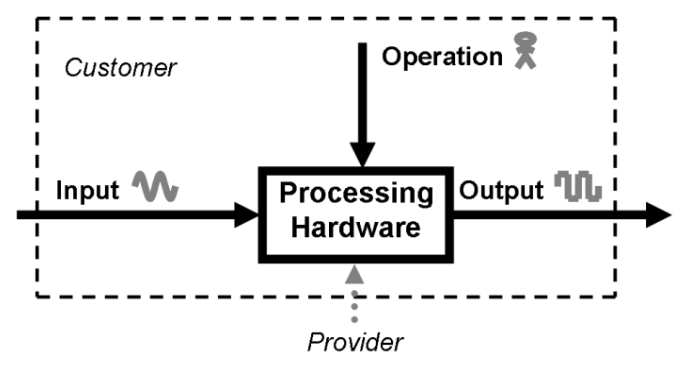

Figure 1. A part of the studied companies' business.

\subsection{Vision and business}

The mining company's vision is to be perceived by the customers as the iron ore and industrial minerals supplier that provides the most added value of "performance in ironmaking", including both products and services.

As a customer, the mining company usually purchases components for their manufacturing line in a "traditional way", i.e. they own the physical artifact after the purchase, which is usually performed in several steps. Services, spare parts and upgrading of the physical artifact are bought as possible addons to the ordinary business agreement or separately, and the mining company is therefore responsible for the physical artifact throughout its lifecycle. However, there exist both major and minor successful examples where the mining company has tried to buy services/functions instead of physical artifacts in their manufacturing line.

The vision for the pulp and paper company is to be the leading knowledge-based, technology company. They aim to improve the productivity and competitiveness of their customers' industrial core processes. The company further states that this shall be achieved by "Growth through partnership - Engineering Customer Success". Currently, the pulp and paper provider is usually only responsible for providing the processing hardware according to an agreement (seen as a dotted line in Figure 1). The ownership of the processing hardware is then transferred from the provider to the customer after necessary conditions are fulfilled, after which a sequential monetary transaction takes place. On delivery, acceptance has to be reached together with the customer. Normally, the acceptance is related to a performance test to secure process guarantees, product quality and capacity. The provider does not alone control all the inputs to reach acceptance, e.g. raw material quality, but the last monetary installment should work as an incentive for both parties to finalize the project as fast as possible. After the contract of acceptance is signed, the customer is responsible for the hardware (production and maintenance) and the guarantee is valid. Now, the customer is responsible for everything inside the dashed line in Figure 1 (input, output operation and the hardware itself). 


\subsection{Product development practices}

The development processes for the mining company can be split into two major parts, the product development and the process development. The former working process aims towards external customers, e.g. the steel industry, where the valueadded iron ore products are the main outcome. The latter working process aims towards the internal customers, i.e. their own production unit and manufacturing line. The different stages of the two working processes are similar and can be described as follows. First, ideas from both internal and external sources are collected and evaluated. Then, a pilot study is done to investigate the performance and feasibility of the initial concept, both from a technical and business point of view. If the pilot study is successful, the concept is further developed and evaluated to its limits in several steps before it is finally prepared and launched for production or commercialization.

For the pulp and paper company, the product development follows a stage gate process. The first stage is called "Idea and Management", where ideas from both internal and external sources are collected and screened against strategy, profitability and growth targets. A plan of study is defined and a resource investor is sought. When that is achieved, a decision is taken at the first gate. In this stage, usually, no simulations are performed. The second stage is called "Feasibility", where the technical and commercial feasibility of the concept is assessed. Measurable targets are further set and a business and project plan is established.

The third stage is called "Research and development". Here, different concepts are generated and evaluated. The main simulations are performed in this stage. The fourth stage is called "Solution development" and, here, the product detailed design is finished and tested. The fifth stage is divided into two sub-stages called "Launch" and "Review". In the Launch stage the marketing and launch plan are implemented and a transfer from project organization to line organization is carried through. In the review-stage the objectives are evaluated and the findings are documented. Entering the fifth gate requires that a final report of the project is written with recommendations for other projects.

\subsection{Simulation methodologies}

The usage of simulation tools in combination with model development in the research and development processes of the processing hardware, at both companies, is becoming more and more important in order to reduce overall cost and the number of design cycles. Simulation tools and approaches are especially important, since full-scale experiments are both expensive and difficult to perform. Current simulations are used to verify the design and qualitatively guide the designer in how to change the product design. This approach may be seen when simulations regarding, for instance, material strength, pressure vessels, bearing lifetime, dynamics and fluid mechanics are performed.
The designer proposes a preliminary solution to a calculation engineer who performs the simulations and brings back the results. If the results are not acceptable, the designers propose new solutions for the calculation engineer until the results are acceptable. Since some of the simulations are time-consuming, each designer-calculation-iteration may take days or even a week to finish.

\section{A PROCESSING FUNCTION TO-BE SCENARIO}

In some industries, new business agreements regarding functions rather than hardware are advancing. For the process industries however, the traditional hardware-purchase business model still takes precedence. Since, in other industries, function contracts are becoming more prevalent, it is of interest for the companies' studies here to investigate different consequences of such offers in their own industries. The interviews and workshops resulted in a to-be scenario that both companies found suitable with respect to their particular processes. Common for the companies studied is that the function of interest is to some extent similar, as stated in the previous section.

In this scenario, a process company (PC) will offer a processing function illustrated in Figure 2. Here, the customers are responsible both for the properties of the raw material entering the processing hardware and the operation, while the function provider is responsible for producing the output material properly (see specification level presented in Figure 3). Thus, the ownership of the hardware providing the functions is kept by the function provider, who charges the customers for the output function.

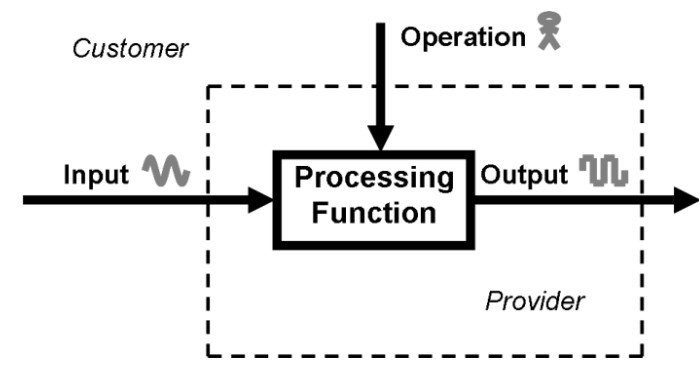

Figure 2. The To-Be scenario.

\subsection{Input specification}

It is critical for the function provider to know the input specifications, since these lead to requirements on the processing function. When developing the hardware enabling the processing function, these requirements should be decomposed into design constraints to be fulfilled. Typical properties for the input specification in both the studied companies are flow rate, pressure, temperatures and 
dimensions. In this scenario, the function contract takes ranges of the input specifications into consideration.

\subsection{Output specification}

The output specification is of main concern for the function customer, without being responsible for how the output specification is realized. This specification would typically consist of performance properties and quality measures. The processing function further has to be conducted in such way that statutory requirements on, for instance, discharges, energy consumption and noise are fulfilled.

\subsection{Operation}

When providing a processing function, as illustrated in Figure 2 , it is not obvious that the operation shall be managed by the function provider. On the contrary, the results from the interviews have shown that both provider and customer wish to keep responsibility for operation with the customer, since the customer usually has better knowledge in this domain. However, this means that the contract must control how the operation shall be performed, since the provider is responsible for the output. This gives an opportunity to create incentives for the operator to run the machines in a cost-effective and sustainable manner, i.e. penalty for sub-optimal operation and benefits for correct operation. Hence, one important objective for the simulations discussed below is to identify suitable ranges for the machine operations.

\section{PROCESSING FUNCTION DECOMPOSITION}

There is no unique solution for getting from the as-is situation to the proposed to-be scenario. However, the function can be decomposed into sub-functions that need to be facilitated by some hardware together with some service and software. Figure 3 shows the processing function decomposition.

There are always aspects to consider when realizing the processing function which are not directly coupled to the facilitating components. These external aspects (e.g. business, political and lifecycle aspects) narrow the design space. However, the external aspects mentioned here are not all conceivable external aspects, rather, they are included here as examples. Business aspects are, for example, budget, strategy and available competencies, while political aspects are things like laws, restrictions, financial support and subventions.
Examples of lifecycle aspects include recycling, waste handling and convertibility of the hardware. Thus, business and political aspects are not seen as sub-sets of lifecycle aspects, since they are external to the supplier's system and something over which he has little control.

The next level consists of three overall sub-domains called process, environment and service. The reason for this particular division is that the process concerns the physical properties of the output, the environment sub-domain concerns external requirements needed to be fulfilled (laws, policies, etc.), while service concerns the provider-related support system required to keep the system operating.

The sub-domain process can further be divided into performance and quality. Performance has to do with the quantity while in this case quality has to do with properties important for the customer value. These measures are still abstract; hence, in order to concretize them, further decomposition has been conducted, as indicated in the figure. Performance, as a part of the process sub-domain, can typically be divided into capacity and availability, whereas quality can be divided into size and strength. These quantities are measurable and therefore a part of the specification of the output building the function. This level is therefore called the specification level.

The environment sub-domain can be decomposed into internal and external environment. To get measurable quantities the internal environment can further be divided into noise and vibrations, while the external environment can be divided into discharges and emissions.

The last sub-domain in the decomposition is service, which is marked with a dashed line, since it is not directly a part of the development of the hardware, but rather an important added value to the hardware-based function. Here, one service is training in how to manage the hardware. As stated in Chapter 1, besides hardware and service, a function also consists of software. The main result presented in this paper is a methodology for simulation-driven design applied on the specification level of Figure 3.

Notice that the model, above the specification level, is meant to be generic for all kinds of functional products of the processing industry which contain hardware, software and service components. Further, the actual hardware enabling output which meets the requirements presented in the specification level is not explicitly shown in Figure 3, since it could be facilitated through several different technical solutions. 


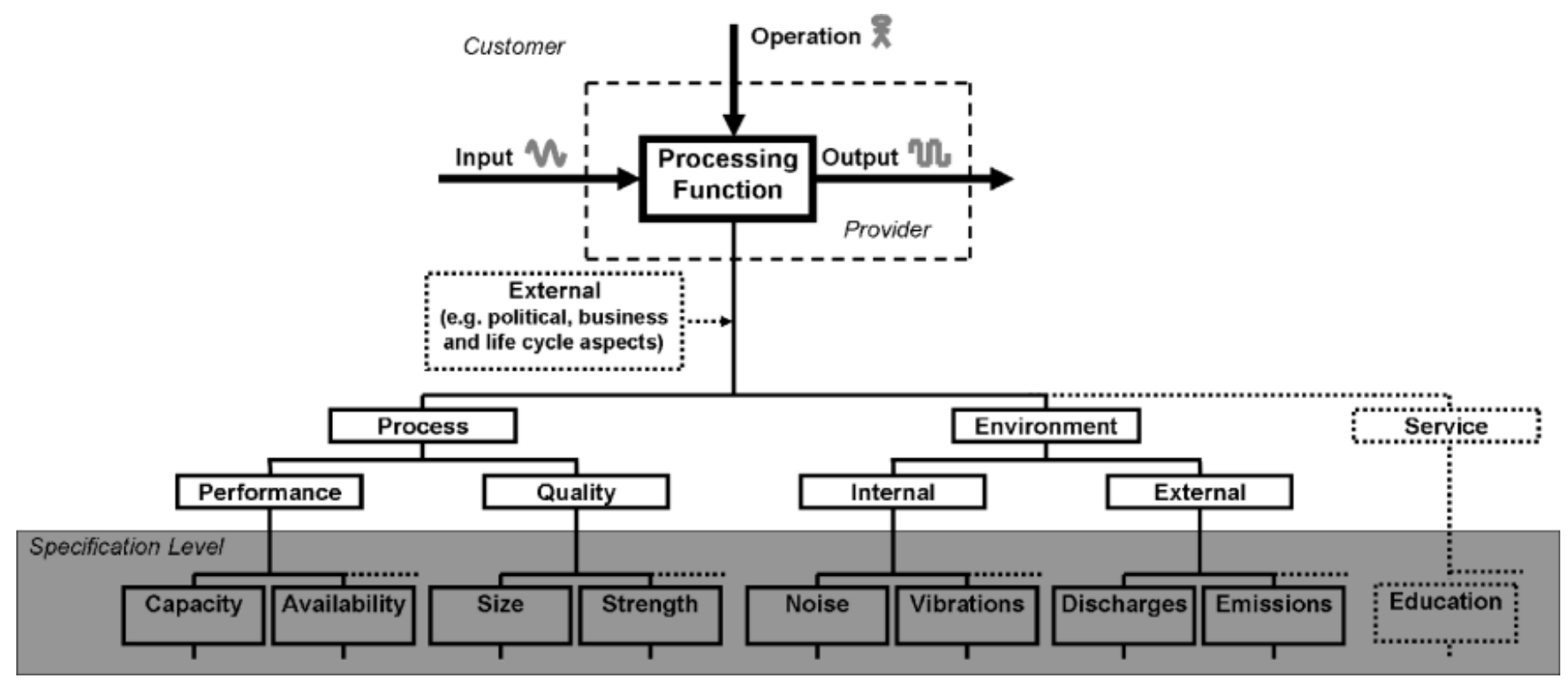

Figure 3. Processing function decomposition.

\section{A METHODOLOGY FOR SIMULATION-DRIVEN DESIGN}

The overall goal for simulations is often to predict profit (by predicting costs and incomes) and performance. Profit and performance often have to be decomposed into some measurable quantities before they can be predicted through modeling and simulation. Therefore, the provider needs to consider every aspect within the dashed line in Figure 2, while everything outside the dashed line has to be considered by the customer. In addition, the provider and customer views are connected and cannot generally be sub-optimized, since the operation and input specification is critical for providing an output within permitted ranges and vice versa. Different situations pose different presumptions for the simulations. Here, the governing processing parameters (excluding input and output parameters) are divided into geometry, operational and external parameters, as shown in Table 1.

The Geometry Parameters category consists of, for example, dimensions and materials, which in general cannot be changed after the hardware that delivers the processing function has been specified or manufactured. Naturally, in certain situations, these parameters are allowed to change after the development process, subject to certain restrictions, as for example during upgrading and/or rehabilitation projects as well as during business offers.
During business offers, for instance, the geometry parameters are often only variable by choosing one hardware solution from a set of discrete hardware concepts and not by varying the geometry parameters of one concept continuously. Hence, design parameters are only variable during the development process, while they can be supposed to be fixed during business offer and operation situations. Hardware operational parameters, such as flows, speeds and temperatures, are highly dependent on the chosen design parameter configuration and are in general variable within some intervals. External parameters, such as political, business and lifecycle aspects, are examples of parameters that usually vary over time (for example, dollar, fuel and electricity rates, emissions rights trading, risks and strategic assessments). Since many of these parameters may change on a daily basis, they are only explicitly known during operation, while certain assumptions have to be made on these parameters both in the development and business situation. A characteristic feature of these parameters is, furthermore, that they often constrain the space from which feasible solutions are extracted rather than affecting the governing equations. The geometry, operational and external parameters together will be referred to as design parameters, and the space spanned by the design parameters as design space. In order to find feasible solutions the target function must be evaluated within the design space. The space spanned by the design parameters and evaluated target functions will further be referred to as solution space. 
Table 1. Considerations for simulations in different situations.

\begin{tabular}{|l|c|c|c|}
\hline \multicolumn{1}{|c|}{ Purpose } & Development & Business Offer & Operation \\
Parameter category & & & \\
\hline Geometry & Variable & Fixed & Fixed \\
\hline Operational & Variable & Variable & Variable \\
\hline External & Unknown & Unknown & Known \\
\hline
\end{tabular}

In order to drive the solutions in a processing-function businessscenario perspective (see Figure 3) we here suggest a methodology which combines developed computer models with standard optimization frameworks to find optimal solutions, instead of using the more traditional "trial and error" procedure (see Chapter 3.1). The herein proposed methodology requires that a relatively large number of design points must be evaluated, meaning that rather fast simulations, i.e. short execution times, are required for the methodology to be effective. However, there are optimization methods for computer-demanding problems as well. Surrogate-based optimization analysis [25] is one such optimization method. It provides an approximate solution space based on a few runs of computational time-consuming virtual models.

The proposed methodology can be divided into the following four key steps:

Model Definition, Problem Scaling, Design Exploration and Design Extraction, as described briefly below. The Design Exploration step is further split in two paths, depending on the degree of the computer model, as shown in Figure 4. The proposed methodology may be used in early stages of the design process. This means that the models used can identify large sets of concept designs to be further evaluated. Thus, the model allows for a relative evaluation between the different concepts.

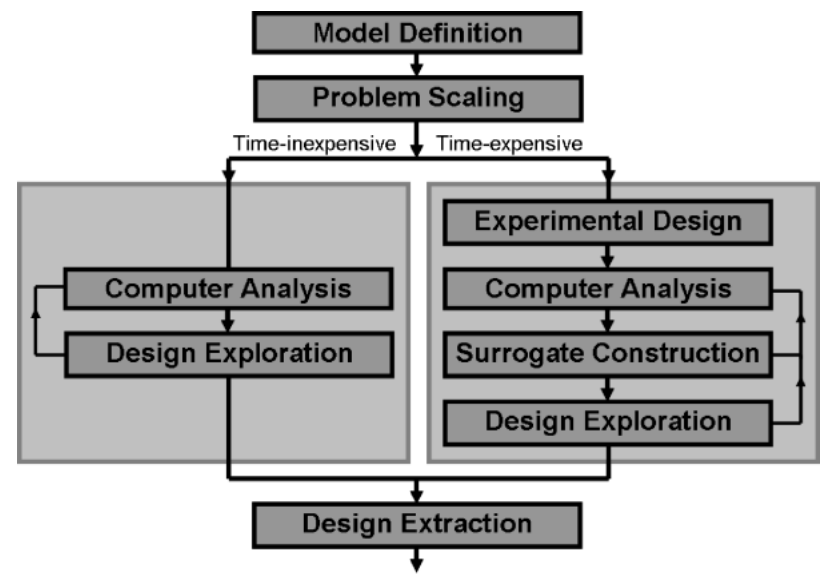

Figure 4. An example of a general flow chart in the proposed methodology, including both computerexpensive and inexpensive problems.

\section{Step 1. Model Definition}

In the first step a computer model which represents the addressed property in the function specification level is defined. The virtual model can describe either a single machine component or several connected machine components in a process line simultaneously, and can either be general or specific as well as high-fidelity or low-fidelity, depending on the problem and business scenario studied. Specifically, a general virtual model represents several different design concepts simultaneously, while a specific model only represents one design concept at a time. This means that general models have a higher level of abstraction than specific models. A highfidelity virtual model has high accuracy and a low-fidelity model has lower accuracy. Hence, high-fidelity models have higher level of detail than low-fidelity models.

\section{Step 2. Problem Scaling}

The simulation and optimization problem can normally be simplified by scaling the corresponding equations, parameters and constraints in the adopted virtual model properly. Scaling results in non-dimensional parameters and variables, often leading to a reduced design space and therefore also often to a reduced total execution time.

\section{Step 3. Design Exploration}

During design exploration, the design space is examined and explored with the aid of the adopted virtual model, as described in steps one and two. Powerful tools for design exploration include optimization, visualizations and sensitivity analysis. In the case of computationally inexpensive virtual models, the complete solution space can be simulated,

For optimization analysis, there are in general two broad classes of analysis tools; gradient-based and non-gradient-based algorithms [26]. For computationally time-demanding problems, this step can further be aided by using a surrogate model approach [25]. Hence, three additional steps, i.e. Experimental Design, Computer Analysis, and Surrogate Model Construction, have to be performed, as indicated in the right 
path in Figure 4. In this first additional step (Experimental Design), a number of prescribed data points are chosen from the design space, according to some experimental design technique [25]. In the second additional step (Computer Analysis), the computer-demanding analysis for each selected data point in the first additional step is evaluated, and in the third and final additional step a surrogate model is fitted, validated and perhaps also updated (if its fidelity is poor) to mimic the behavior of the computer analysis in the second additional step. Then, by using this surrogate model instead of the original virtual model in this mutual Design Exploration step, the total computational load can be substantially reduced as well as allowing designers to gain a better insight into the design and solution space characteristics such as the interplay and sensitivity between the design variables and objective functions. However, the outcome of the surrogate-based optimization analysis is highly dependent on the effectiveness of the adopted surrogate model(s).

\section{Step 4. Design Extraction}

A crucial step in the previous Design Exploration step is the selection of appropriate parameters, objective functions and constraints which span the design space, since they significantly affect the load and outcome of the optimization problem. In most situations, it is almost impossible to optimize all objectives simultaneously; instead, we must adopt compromises or trade-offs, e.g. between profit and performance. In principle, there are usually many equally good solutions, known as Pareto optimal solutions. Generally, the choice depends on whether we want a cheap, low-performance machine component or an expensive, high-performance one, or something in-between. In addition, each Pareto optimal solution can itself contain several solutions if a general virtual model is used. Hence, there is a need for the design engineers to extract the "best" overall design point from several concepts and/or Pareto optimal solutions, something which today is often done on the basis of experience and personal standpoints.

\subsection{Example of the proposed methodology}

In order to concretize the proposed methodology, one example from a provider's perspective will be given below. In this case, a rotating machine shall be developed with the purpose to enable a processing function as decomposed in Figure 3. In order to decrease the risk in this agreement, simulations shall be used to drive the design to suitable solutions. The inexpensive path for the design exploration shown in Figure 4 will further be used in this example.

\section{Step 1. Model Definition}

In order to enable a processing function, performance has been indicated to be important, since the maintenance and other services, for instance, which in traditional business give income, are now an expense. In this example, rotordynamics is critical for the performance and hence, a general virtual model for rotordynamics [27] is used (see Figure 5). This model represents fundamentally different concept designs such as overhang rotors, Jeffcott rotors, different types of bearing assemblies and supporting structures, which are all running below the first two forward and backward whirl frequencies.
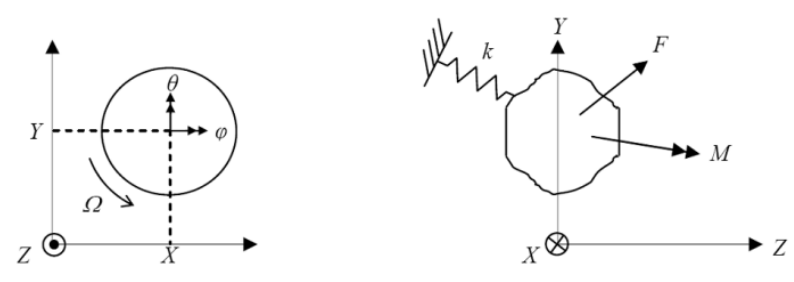

Figure 5. Example of a virtual model.

The corresponding equation of motion Equation (1) contains eight positive numbered parameters $\left(m, J_{t}, J_{p}, \gamma\right.$ and $\left.k_{1}-k_{4}\right)$ where $\mathrm{m}$ is the mass of the rigid body, $J_{t}$, the transversal-,$J_{p}$ the polar principal moment of inertia, $\Omega$ the constant spin speed and $\gamma$ the eccentricity (distance from the geometrical center to the center of gravity). A general way of describing the isotropic stiffness the rotor experiences are by four parameters $k_{1}-k_{4}$ as shown in Equation (1). Loading due to surrounding exposures are denoted $F$ for forces and $M$ for moments as function of time (see Figure 5).

$$
\begin{aligned}
& {\left[\begin{array}{cccc}
m & 0 & 0 & 0 \\
0 & m & 0 & 0 \\
0 & 0 & J_{t} & 0 \\
0 & 0 & 0 & J_{t}
\end{array}\right]\left\{\begin{array}{c}
\ddot{X} \\
\ddot{Y} \\
\ddot{\varphi} \\
\ddot{\theta}
\end{array}\right\}+\Omega\left[\begin{array}{cccc}
0 & 0 & 0 & 0 \\
0 & 0 & 0 & 0 \\
0 & 0 & 0 & J_{p} \\
0 & 0 & -J_{p} & 0
\end{array}\right]\left\{\begin{array}{c}
\dot{X} \\
\dot{Y} \\
\dot{\varphi} \\
\dot{\theta}
\end{array}\right\}+} \\
& {\left[\begin{array}{cccc}
k_{1} & 0 & 0 & -k_{2} \\
0 & k_{1} & k_{2} & 0 \\
0 & k_{3} & k_{4} & 0 \\
-k_{3} & 0 & 0 & k_{4}
\end{array}\right]\left\{\begin{array}{c}
X \\
Y \\
\varphi \\
\theta
\end{array}\right\}=\left\{\begin{array}{c}
m \gamma \Omega^{2} \cos (\Omega t)+F_{X}(t) \\
m \gamma \Omega^{2} \sin (\Omega t)+F_{Y}(t) \\
M_{X}(t) \\
M_{Y}(t)
\end{array}\right\}}
\end{aligned}
$$

\section{Step 2. Problem Scaling}

For the illustrative example, [27] showed that introducing the nondimensional (marked with a ${ }^{* *}$ ) d.o.f. and time,

$$
\begin{aligned}
& X^{*}=X / \gamma, Y^{*}=Y / \gamma, \varphi^{*}=k_{2} \varphi / m \gamma \Omega^{2}, \\
& \theta^{*}=k_{2} \theta / m \gamma \Omega^{2} \text { and } \tau^{*}=\Omega t
\end{aligned}
$$

into Equation (1) gives, 


$$
\begin{aligned}
& {\left[\begin{array}{llll}
1 & 0 & 0 & 0 \\
0 & 1 & 0 & 0 \\
0 & 0 & 1 & 0 \\
0 & 0 & 0 & 1
\end{array}\right]\left\{\begin{array}{l}
\ddot{X}^{*} \\
\ddot{Y}^{*} \\
\ddot{\varphi}^{*} \\
\ddot{\theta}^{*}
\end{array}\right\}+\Omega\left[\begin{array}{cccc}
0 & 0 & 0 & 0 \\
0 & 0 & 0 & 0 \\
0 & 0 & 0 & J^{*} \\
0 & 0 & -J^{*} & 0
\end{array}\right]\left[\begin{array}{l}
\dot{X}^{*} \\
\dot{Y}^{*} \\
\dot{\varphi}^{*} \\
\dot{\theta}^{*}
\end{array}\right\}+\left[\begin{array}{cccc}
k_{1}^{*} & 0 & 0 & -1 \\
0 & k_{1}^{*} & 1 & 0 \\
0 & k_{3}^{*} & k_{4}^{*} & 0 \\
-k_{3}^{*} & 0 & 0 & k_{4}^{*}
\end{array}\right]\left\{\begin{array}{l}
X^{*} \\
Y^{*} \\
\dot{\varphi}^{*} \\
\theta^{*}
\end{array}\right\}} \\
& =\left\{\begin{array}{c}
\cos \left(\tau^{*}\right)+F_{X}^{*}\left(\tau^{*}\right) \\
\sin \left(\tau^{*}\right)+F_{Y}^{*}\left(\tau^{*}\right) \\
M_{X}\left(\tau^{*}\right) \\
M_{Y}\left(\tau^{*}\right)
\end{array}\right\}
\end{aligned}
$$

The nondimensional geometry and operational parameters then become,

$$
\begin{aligned}
& J^{*}=J_{p} / J_{t}, k_{1}^{*}=k_{1} / m \Omega^{2}, k_{3}^{*}=k_{2} k_{3} / m J_{t} \Omega^{4} \\
& k_{4}^{*}=k_{4} / J_{t} \Omega^{2}
\end{aligned}
$$

and the external loading,

$$
\begin{aligned}
& F_{X}^{*}\left(\tau^{*}\right)=\frac{F_{X}\left(\tau^{*} / \Omega\right)}{m \gamma \Omega^{2}}, F_{Y}^{*}\left(\tau^{*}\right)=\frac{F_{Y}\left(\tau^{*} / \Omega\right)}{m \gamma \Omega^{2}}, \\
& M_{X}^{*}\left(\tau^{*}\right)=\frac{k_{2} M_{X}\left(\tau^{*} / \Omega\right)}{m J_{t} \gamma \Omega^{4}}, M_{Y}^{*}\left(\tau^{*}\right)=\frac{k_{2} M_{Y}\left(\tau^{*} / \Omega\right)}{m J_{t} \gamma \Omega^{4}}
\end{aligned}
$$

The number of parameters in the problem has now been reduced from nine to four.

\section{Step 3. Design Exploration}

The geometry and operational parameters are defined by Equation (8)-(11) and in this paper

$$
r^{*}=\left(\sqrt{\left(X^{*}\right)^{2}+\left(Y^{*}\right)^{2}}\right)_{\max }
$$

the maximum nondimensional distance between the geometrical center and the $Z$-axis is used as the objective function. Keeping $J^{*}$ gives a design space $\mathrm{D}_{\mathrm{s}} \in \mathfrak{R}^{4}$, which can be represented in three-dimensional plots where the axes are given by three remaining parameters and the color shows the value of the objective function in every design point. This is of course a volume containing different colors, and in order to see the colors in the 'solid', slices, discrete values of the parameters are visualized. The responses depend on what type of load the rotor is exposed to and in this study, an impulse will be considered.
Figure 6 shows the $D_{s}$ for $J^{*}=0.5$ when the rotor has been subjected to an impulse in the $X$-direction. Transparent areas indicate that the objective function grows exponentially towards infinity (unstable volume). Dark gray is high vibration amplitudes, while light gray represents low vibration amplitudes $r^{*}$.

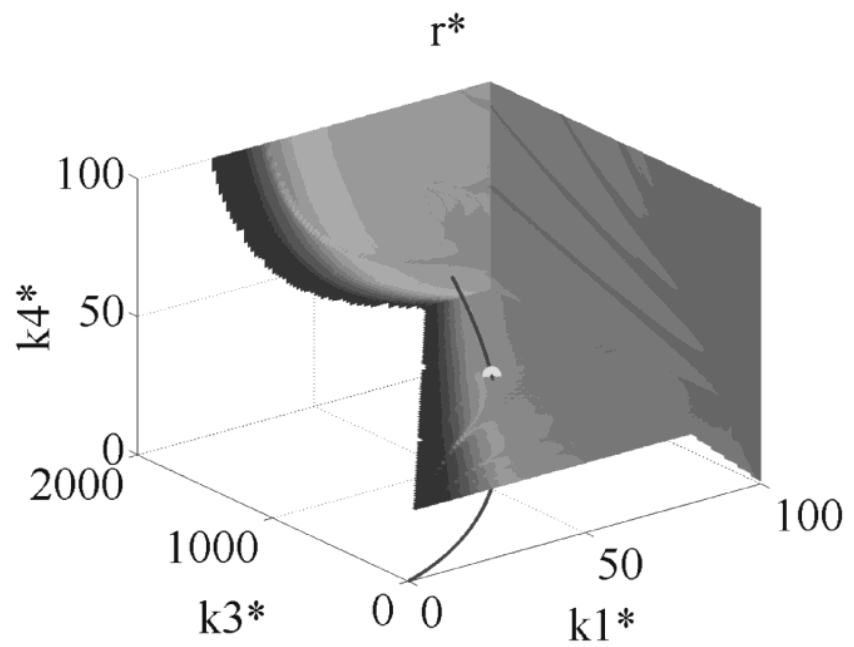

Figure 6. Solution space Ds for an impulse in X-direction when $\mathrm{J}^{*}=0.5$.

Unstable vibrations cannot be accepted when designing rotor dynamical systems; hence, the transparent volume in Figure 6 must be excluded when deriving design points. An optimization algorithm can be applied to find optimum solutions from the remaining solution space. In Figure 6 one design point is illustrated as a light grey dot, where the dark grey line (passing through the light grey dot) represents how this design point changes with spin speed. Note that suitable ranges for the design exploration depend on the type of business agreement. Domains that are valid when trading hardware can even be inappropriate in function business situations. This means that the business situation must be decomposed into ranges and rules for the optimization procedure in order to extract suitable design points.

\section{Step 4. Design Extraction}

The solution point in Figure 6 (light grey dot) can be fulfilled by fundamentally different concepts, see Figure 7. 

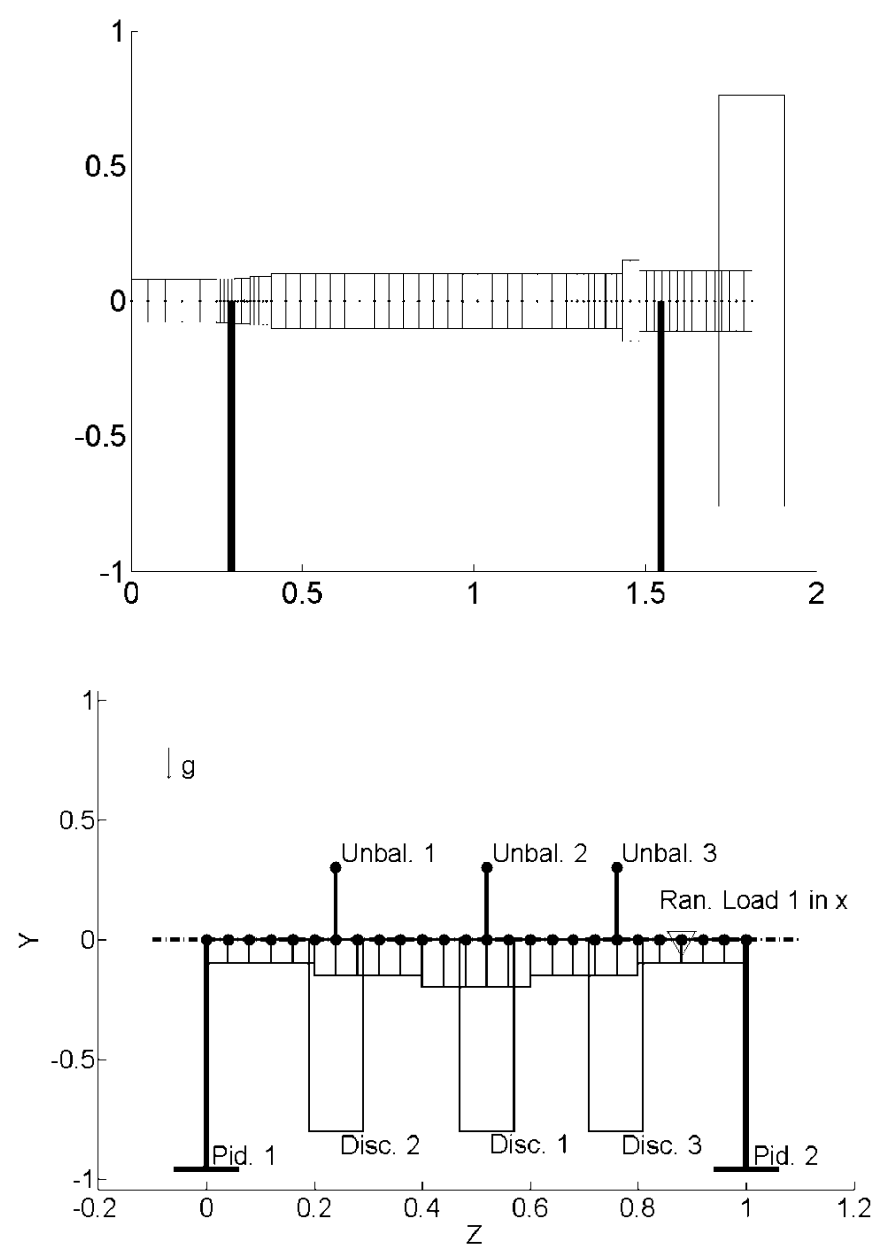

Figure 7. Different concepts fulfilling the design point.

However, the idea here is to force the designers to only use concepts which fulfill the design point(s). For instance, this can be achieved in such way that the designers suggest a design and then get feedback from software which runs the herein presented methodology regarding how certain parameters must change in order for the solution to be valid.

\section{DISCUSSION AND CONCLUSIONS}

For processing businesses, going from traditional hardware trading described in the as-is situation (see Figure 1) to function offers described in the to-be scenario (see Figure 2), affects the responsibilities of the provider and customer. By comparing Figures 1 and 2 it can be seen that in the described processingfunction business scenario the provider responsibilities increase while the customer responsibilities decrease. For simulation methodologies, this means that different constraints have to be managed in procurement compared to provision situations.

Decomposing the processing function (see Figure 3) results in a specification level where, ideally, it should be possible to simulate all properties simultaneously. Changing the business does not affect the physical behavior the simulations predict. However, it affects the domains of feasible solutions to choose among such as constraints, boundary conditions, limitations in solution parameters and objective functions. This means that the proposed methodology can also be used in traditional business situations, although different presumptions for the simulations will hold.

Given the to-be scenario described above, three main situations have been identified where simulations can be used, development, business offer and operation. In this scenario and using the proposed methodology it is plausible that suitable operation ranges may be found through simulations, keeping measurable output (for example, emissions, size, strength, etc.) within agreed upon levels. Thereby, the customer and supplier have more information available earlier than before on which to make decisions and negotiate production and business agreements. Hence, the customer and supplier value may be optimized towards a win-win situation through evaluation and prioritization.

\subsection{The development situation}

For the providers, when developing a processing function as shown in Figure 3, it should be possible to simulate all properties in the specification level as early as in the concept phase. Besides, the simulations should here be used as a predictive tool rather than as a verifying tool after development of a physical prototype. The simulations therefore drive the design process and are not simply used to choose between different proposed designs. By the proposed methodology it is possible to derive a set of valid designs already in the concept phase of the product development process that all meet the specified requirements. As shown in Table 1, in the development situation, both geometry parameters and operational parameters are variable, (at least in the early stages of the product development). External aspects are generally not known and therefore require forecasting in order to be included.

\subsection{The business offer situation}

The proposed methodology can also be used by providers and customers to evaluate and prioritize business offers as well as activities in the business offer process, especially in the context of business-to-business relations during development of functional products [3]. As Table 1 shows, in the business offer situation the geometry parameters and operational parameters are often known, since existing machines are usually considered. However, the operational parameters can be tuned to fit the specific customer's needs. This means that in order to optimize the profit in a business situation, the solutions are restricted to variations in operational parameters and different discrete design parameters (since there may be several machines to consider). As for the development situation, the external 
aspects are in general not known when delivering a business offer. However, time has elapsed since the machines were developed and hence the knowledge base used to forecast the impact of these aspects on the profit has increased.

\subsection{The operation situation}

When the business agreement is signed, the responsibility for operation must be assigned to either the customer or the provider. In the to-be scenario described above, the customer is responsible for the operation. The function provider is, of course, also concerned with the operation, which is further regulated by the terms of the contract. During operation, the geometry parameters are even more difficult to change than in the business offer situation, since one or many machines are installed and running. The operational parameters, on the other hand, are accessible and can hence be used for optimization purposes. In this scenario and using the proposed methodology it is plausible that suitable operation ranges may be found through simulations, keeping measurable output (for example, emissions, size, strength, etc.) within agreed upon levels.

External aspects are further known in the operation situation (at least for the moment). In this situation, the methodology can hence be used to compensate for, e.g. poor forecasting regarding the external aspects or other changes which affect profit.

\section{ACKNOWLEDGEMENTS}

Asc. Prof. Magnus Karlberg, Ass. Prof. Magnus Löfstrand and Prof. Staffan Lundström gratefully acknowledge the contribution from the Swedish Governmental Agency for Innovation Systems (VINNOVA) for funding The Faste Laboratory, Centre for Functional Product Innovation at Luleå University of Technology.

Furthermore, Dr. Magnus Löfstrand also acknowledges The Swedish Foundation for Strategic Research for the SSPI project (Scalable search of Product Lifecycle Information).

\section{REFERENCES}

[1] Rasgado, T., Thompson, G., Elfström, B-O., (2004). 'The design of functional (total care) products'. Journal of Engineering Design, Vol. 15, No. 6, pp. 515-540.

[2] Brännström, O., Elfström, B-O., Thompson, G., (2001). 'Functional products create new demands on product development organisations'. ICED 01, Glasgow, August 21-23. [3] Karlsson, L., Löfstrand, M., Larsson, A., Larsson, T., Törlind, P., Elfström, BO., Isaksson, O., (2005). 'Information driven collaborative engineering: Enabling functional product innovation'. The $3^{\text {rd }}$ international workshop on challenges in collaborative engineering, CCE05, Sopron, Hungary, April 1315.

[4] Löfstrand, M., Larsson, T., Karlsson, L., (2005). 'Demands on engineering design culture for implementing functional products'. International conference on engineering design, Melbourne, Australia, August 15-18.

[5] Kim, S. H. (2007). 'Performance contracting in after-sales service supply chains'. Management Science 53(12): 1843.

[6] Eckert, C. M., R. Keller, Earl, C., Clarkson, P. J., (2006). 'Supporting change processes in design: Complexity, prediction and reliability'. Reliability Engineering \& System Safety 91(12): 1521-1534.

[7] Terry, M., Mynatt, E. D., (2005). 'Enhancing generalpurpose tools with multi-state previewing capabilities', Knowledge-Based Systems 18(8): 415-425.

[8] Jackson, C., (2006). 'The Simulation-Driven Design Benchmark Report: Getting It Right the First Time', Obtained through the internet: http://www.aberdeen.com/summary/report/benchmark/BM _Simulation_driven_Design_3591.asp, requires free registration, [Accessed 18/4/2008].

[9] Roosenburg N.F.M., and Eekels J., (1995). Product Design: Fundamentals and Methods, USA, John Wiley and Sons.

[10] Becker, M.C., Salvatore, P., and Zirpoli, F., (2005). 'The impact of virtual simulation tools on problem-solving and new product development organization', Research Policy, Vol. 34, No. 9, pp. 1305-1321.

[11] Sinha, R., Paredis, C., J.J., Liang, V-C., and Khosla, P., K., (2001). 'Modeling and simulation methods of engineering systems', Journal of computing and information science in engineering 1, 84-91.

[12] Sellgren, U., (1995). 'Simulation driven design, A functional view of the design process'. Licentiate thesis. Royal Institute of Technology, Sweden.

[13] Rova, J-E., (1998), 'Simulation-driven-design leads to world's lightest two-seat floatplane', Scientific computing and automation, Vol. 15, No. 11, pp. 35-37.

[14] Makkonen, P., (1995). On Multi body systems simulation in product design. $\mathrm{PhD}$ thesis. Royal institute of technology, Sweden. 
[15] Larsson, T., (2001). Multibody dynamic simulation in product development. $\mathrm{PhD}$ thesis. Luleå University of Technology, Sweden.

[16] Filla, R., and Palmberg, J-O., (2003). 'Using dynamic simulation in the development of construction machinery', The Eighth Scandinavian International Conference on Fluid Power, SICFP'03, Tampere, Finland, May 7-9.

[17] Angantyr, A., and Aidanpää, J-O., (2004). 'A Pareto based generic algorithm search approach to handle damped natural frequency constraints in turbo generator rotor system design'. ASME journal of engineering for gas turbines and power, 126, 619-625.

[18] Marjavaara, B.D., Lundström, T.S., Goel, T., Mack, Y., Shyy, W., (2007), 'Hydraulic Turbine Diffuser Shape Optimization by Multiple Surrogate Model Approximations of Pareto Fronts', Journal of Fluids Engineering, Vol. 129, No. 9, pp. 1228-1240.

[19] Alonso, J. J., LeGresley, P., Pereyra, V., (2009). 'Aircraft design optimization'. Mathematics and Computers in Simulation 79(6): 1948-1958.

[20] Starling, A. C., Shea, K. (2005). 'Virtual Synthesisers for Mechanical Gear Systems'. International conference on engineering design, Melbourne, Australia, August 15-18.

[21] Bylund, N., (2004). Simulation driven product development applied to car body design, $\mathrm{PhD}$ thesis. Luleå University of Technology, Sweden.

[22] Wall, J., (2007). Simulation-driven-design of complex mechanical and mechatronic systems, $\mathrm{PhD}$ thesis. Blekinge Institute of Technology, Sweden.

[23] Löfstrand, M., (2007). A modelling and simulation approach for linking design activities to business decisions, PhD thesis, Luleå University of Technology, Sweden.

[24] Yin, R.K., (1994). Case Study Research, Design and Methods, USA, Sage publications.

[25] Simpson, T.W., Booker, A.J., Ghosh, D., Giunta, A.A., Koch, P.N., Yang, R.-J., (2004), 'Approximation methods in multidisciplinary analysis and optimization: A panel discussion', Structural and Multidisciplinary Optimization, Vol. 27, No. 5, pp. 302-313.

[26] Rao, S.S., (1996), Engineering Optimization: Theory and Practice, John Wiley and Sons, New York.

[27] Karlberg, M. and Aidanpää, J-O., (2002). 'Evaluation of rotordynamical concepts subjected to rotating unbalance and impulse'. Proceedings of the Sixth International Conference on Rotor Dynamics, Sydney, Australia, 2, pp. 752-758. 